\title{
Glauco Sanga, La fiaba: morfologia, antropologia e
} storia

Cluep, Padova, 2020

Antonio Fanelli

\section{CpenEdition}

\section{Journals}

Edizione digitale

URL: http://journals.openedition.org/aam/3686

DOI: $10.4000 /$ aam.3686

ISSN: 2038-3215

Editore

Dipartimento Culture e Società - Università di Palermo

Notizia bibliografica digitale

Antonio Fanelli, «Glauco Sanga, La fiaba: morfologia, antropologia e storia», Archivio antropologico mediterraneo [Online], Anno XXIII, n. 22 (2) | 2020, online dal 31 décembre 2020, consultato il 27 janvier 2021. URL: http://journals.openedition.org/aam/3686 ; DOl: https://doi.org/10.4000/aam.3686

Questo documento è stato generato automaticamente il 27 janvier 2021.

\section{$\oplus \Theta \Theta \Theta$}

Archivio antropologico mediterraneo è distribuita con Licenza Creative Commons Attribuzione - Non commerciale - Non opere derivate 4.0 Internazionale. 


\section{Glauco Sanga, La fiaba: morfologia, antropologia e storia}

Cluep, Padova, 2020

Antonio Fanelli

NOTIZIA

GLAUCO SANGA, La fiaba: morfologia, antropologia e storia, Cluep, Padova, 2020.

ISBN 9788854952805 
1 Il libro di Glauco Sanga riporta al centro dei nostri studi uno dei più blasonati tra $\mathrm{i}$ generi della letteratura folklorica, sin dall'epoca romantica segnata del successo internazionale dell'opera fiabistica dei fratelli Grimm e dalla esaltazione dell'oralità popolare come matrice autentica dell'arte e dell'identità nazionale. Nella stagione positivista vi fu una grande fioritura di ricerche sulla fiaba, anche in Italia, grazie a Giuseppe Pitrè, Domenico Comparetti e Vittorio Imbriani. Inoltre, nel nostro paese, il fervore documentario della demologia gramsciana nel corso degli anni '60 e '70 alimentò un altro momento di particolare visibilità per gli studi sulla narrativa orale grazie alla monumentale raccolta di fiabe

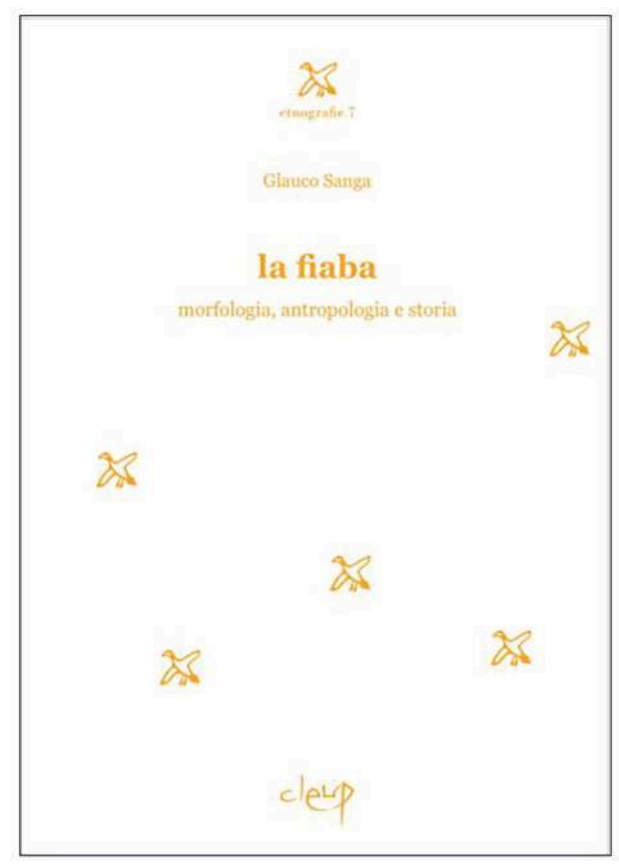
in tutte le regioni italiane per conto della

Discoteca di Stato, condotta da una vasta équipe con la supervisione di Alberto Mario Cirese. Non mancarono poi ulteriori sviluppi della fiabistica, grazie all'opera di Aurora Milillo e del gruppo di ricerca della Regione Lombardia coordinato da Roberto Leydi, e vi furono diversi convegni internazionali di carattere interdisciplinare a incrementare attorno al tema della fiaba lo scambio tra folkloristi, letterari e semiologi. Mentre le forme di documentazione magnetofonica si affinavano e le riflessioni sulle trascrizioni fonetiche offrivano maggiori spazi di fedeltà alla performance orale e al valore degli interpreti, la fiabistica cominciò lentamente a declinare nei due decenni successivi. Infatti, i generi della letteratura orale vennero relegati ai margini dai nuovi approcci critici e decostruttivi che irruppero nel campo antropologico segnando un momento di frattura netta con gli studi precedenti. Pertanto, questo volume copre innanzitutto un vuoto in ambito antropologico, offrendo uno strumento utile ed esaustivo per la didattica universitaria dove sono decisamente scarsi i testi di inquadramento generale sulla fiaba.

2 L'opera nasce dalla lunga esperienza di Glauco Sanga come studioso dell'oralità tradizionale in grado di coniugare in maniera originale la dialettologia con gli studi demo-etno-antropologici, grazie al magistero veneziano e alla direzione di due riviste innovative per lo sviluppo dell'antropologia culturale nel nostro paese (la «Rivista Italiana di Dialettologia» e, soprattutto, «La Ricerca Folklorica» che sin dagli anni '80 ha favorito il dibattito nell'antropologia italiana). Sanga si pone in modo chiaro su un asse teorico-interpretativo di tipo morfologico, basato sulla centralità della analisi propperiana delle fiabe. Il libro è organizzato in dieci capitoli, con una ampia introduzione sui temi dell'oralità e della cultura popolare (capitoli 1 e 2), a cui seguono i cinque capitoli centrali del libro (dal 3 al 7) dedicati alla fiaba e alla disamina delle teorie di Propp, con un particolare approfondimento della vicenda editoriale della sua opera e della ricezione e traduzione in Italia (assolutamente pionieristica e originale, visto che Le radici storiche dei racconti di fate del 1946 apparvero in Italia già nel 1949 nella «collana viola» di Einaudi, con largo anticipo sulle traduzioni in altre lingue). 
Ampio spazio è dedicato al celebre dibattito fra Lévi-Strauss e Propp su strutturalismo e formalismo, e questa sezione si chiude con una vera e propria riconfigurazione del modello di analisi morfologica grazie ad alcune proposte di integrazione e di modifica del modello proppiano. Infine, il libro presenta tre capitoli che ripercorrono gli studi sulla fiaba basati sulla Filologia (cap. 8), l'Etnografia (cap.9) e l'Antropologia (cap.10). Oltre a enucleare la genealogia intellettuale che ha scandito la fiabistica dall'Ottocento ad oggi, l'autore si addentra in una minuziosa disamina del dibattito sulla genesi delle fiabe e propone, anche in questo caso, una tesi originale.

In apertura, come già accennato, vi è un ampio spazio dedicato ai prolegomeni della fiaba, ossia l'oralità, la memoria culturale e la cultura popolare. L'autore apre il volume con un mito di fondazione del distacco siderale tra oralità e scrittura e tra cultura letteraria e oralità folklorica, con la novella di Sacchetti sulla diatriba fra Dante Alighieri e un fabbro fiorentino che vale la pena rievocare. Il vate della letteratura ascoltando i suoi versi intonati da un fabbro con evidenti adattamenti folklorici e modifiche dei versi dovute alla trasmissione orale del testo si era accanito contro i suoi attrezzi scaraventandoli a terra. Per motivare il suo gesto di fronte al fabbro attonito, il poeta aveva esclamato: «Tu canti il libro e non lo di' com'io lo feci, io non ho altr'arte e tu me la guasti» (p.15). Si tratta di un topos letterario presente già nella Grecia antica $\mathrm{e}$ nello stesso periodo di Dante, anche in Spagna, nell'opera di Juan Manuel. Il punto chiave è la distinzione netta tra opera originale, autore e proprietà letteraria che si instaura fra i ceti colti rispetto alla impersonalità del mezzo orale, creando così uno iato tra i due sistemi di comunicazione. La vicinanza con il volgo e con le forme orali di trasmissione della cultura era avvertita come pericolosa e la volontà di creare una netta distinzione verso il basso aprì ad una plurisecolare svolta elitaria fra gli uomini di cultura. Nonostante le forme di circolazione culturale operanti anche nei secoli successivi sarà il Romanticismo a rovesciare questo assunto spingendo gli artisti e i letterati verso la ricerca della matrice autentica della cultura (e dell'identità nazionale) fra gli uomini e le donne illetterate del mondo rurale, pastorale e della montagna. Se la scrittura consente una forma di coscienza storica e la creazione di norme e di vincoli con valenza generale, l'oralità conserva altre virtù come quelle legate alla complessità dei «pronomi personali» e alla fluidità delle genealogie analizzate da Jack Goody. Nei capitoli iniziali si riserva, inoltre, ampio spazio alle teorie classiche di Pëtr Bogatyrëv e Roman Jakobson sulla «sanzione collettiva» come forma di regolazione dell'oralità folklorica e agli approcci strutturalisti nella linguistica introdotti da Ferdinand Saussurre, con la cruciale distinzione tra langue e parole. Viene poi esplorata la stratificazione sociale dei ceti popolari, con un particolare richiamo agli studi postbellici ispirati a Gramsci e alla varietà di contesti sociali delle classi popolari al di là dell'ambito rurale-contadino. In più passi di questo ampio discorso introduttivodidattico viene sottolineato un aspetto cruciale della fiabistica, la traduzione dall'oralità alla scrittura attraverso la trascrizione-riscrittura delle fiabe e, soprattutto, l'aspetto dialogico sia della trasmissione orale della fiaba sia della ricerca etnografica, un tema emblematico che verrà ripreso alla fine del volume.

4 Ma vediamo meglio l'analisi dell'opera di Propp che resta il punto di riferimento cruciale per il libro di Sanga. La teoria morfologica era fino a pochi anni fa un elemento chiave della formazione antropologica ma negli ultimi tempi la centralità di Propp $\mathrm{e}$ delle teorie morfologico-strutturaliste è stata decisamente accantonata e per questo motivo è utile sintetizzarla seguendo l'opera di Sanga. L'analisi morfologica non si rifà alle indagini comparative di taglio diffusionistico della scuola finnica, incentrate sulla 
classificazione delle fiabe per «tipi» (racconti compiuti, autonomi e autosufficienti) e «motivi» (unità narrative minime) raggruppati negli indici internazionali stilati da Antti Aarne e poi perfezionati dal folklorista americano Stith Thompson (e in seguito da Hans-Jörg Uther). Il punto di svolta nell'opera di Vladimir J. Propp è lo studio delle fiaba attraverso le «funzioni». Nella narrativa popolare vi sono alcuni generi canonici, come la favola, la novella, le storielle e le barzellette, le leggende e i racconti. Nonostante la porosità e la fluidità delle categorizzazioni del flusso narrativo Sanga ritiene utile operare questa preliminare distinzione per esaminare quella porzione di patrimonio culturale tramandato oralmente che ha un carattere magico $\mathrm{e}$ soprannaturale, uno schema finzionale e non realistico.

Indicando con «favola» esclusivamente le favole di animali, quelle infantili e i racconti a catena, sulla scia di Propp, anche Sanga adopera il termine «fiaba» per riferirsi alle «fiabe magiche», al cui interno vi è la sotto-categoria delle «fiabe d'incantesimo». Le prime hanno per protagonista un eroe cercatore, di solito maschio, mentre le seconde presentano una figura chiave femminile, un eroe vittima, di solito una fanciulla perseguitata. Se lo studio degli intrecci e dei motivi ha permesso di individuare generi, specie e varietà di fiabe, producendo un utile repertorio basato su una classificazione esteriore, è rimasto eluso, fino al lavoro di Propp, il problema della evidente somiglianza delle fiabe su tutto il globo. Questo dato empirico, vista l'impossibilità e la improbabilità di stabilire dei nessi causali e dei contatti effettivi tra le popolazioni, ha messo in crisi le ipotesi diffusioniste. Pertanto, invece di chiedersi dove abbia origine una fiaba la domanda da porsi è in che cosa consiste una fiaba e quale sono le leggi che determinano la regolarità della narrazione fiabistica. Propp trova la soluzione nello studio morfologico delle «funzioni dei personaggi» poiché le fiabe pur sembrandoci diverse sono in realtà, in qualche modo, sempre la stessa fiaba poiché cambiano i nomi e gli attributi dei personaggi ma le loro azioni sono identiche. Infatti, scrive Propp ne $L a$ morfologia della fiaba (1928, tradotto in Italia a cura di G. L. Bravo per Einaudi nel 1966): «Le funzioni sono straordinariamente poche e i personaggi straordinariamente numerosi. Ciò spiega l'ambivalenza della favola: la sua sorprendente varietà, la sua pittoresca eterogeneità, da un lato, e la sua non meno sorprendente uniformità e ripetibilità dall'altro». La funzione viene identificata dalla sua collocazione nello svolgimento del racconto e nelle fiabe di magia si ritrova una omologia strutturale delle narrazioni, una sorta di monotipo replicabile e scomponibile in infinite varianti, grazie al meccanismo delle iterazioni, di regola delle tripartizioni degli elementi variabili della fiaba, adoperate per fini artistici e performativi. Propp si distacca dall'approccio storico-diffusionistico poiché dà ragione delle omologie e delle somiglianze ipotizzando che lo stesso elemento culturale possa avere origine indipendentemente dalle relazioni e dai contatti storici presso popolazioni che presentano la medesima struttura sociale, economica e produttiva. Propp diviene in tal modo un precursore degli approcci strutturalisti e Lévi-Strauss gli riconobbe il merito di aver colto la replicabilità della fiaba alla ricerca di meccanismi di regolarità soggiacenti alle singole narrazioni. Ma lo studioso francese ingaggiò anche un serrato dibattito col maestro russo distinguendo lo strutturalismo dal formalismo, riconobbe a Propp un ruolo maieutico ma lo confinò al rango di maestro che percorre una via sbagliata. Per affrontare questa complessa sfida epistemologica Lévi-Strauss ricorse a una sorprendente analogia tra vasi e corpi, dove i primi possono contenere di tutto (acqua, olio, olive, etc.), mentre i secondi sono involucri non generici e amorfi poiché indissolubili dal contenuto di ogni sua parte. Pertanto i formalisti, come Propp, incappavano nell'errore di occuparsi della forma 
esteriore, mentre lo strutturalismo puntava a cogliere la forma interna, il contenuto e le sue leggi.

6 Nel caso di Propp questo scivolamento è dovuto alla fluidità delle fiabe mentre una efficace analisi strutturalista si può ottenere, per Lévi-Strauss, con lo studio dei miti. Il «miraggio formalista» si deve, secondo Lévi-Strauss, alla ossessione per le spiegazioni delle origini storiche mentre il problema cruciale nello studio delle fiabe resta quello del contesto etnografico. La replica di Propp fu veemente e allo studioso francese fece rilevare, innanzitutto, che la sua analisi della fiabe si basava su materiali frutto di ricerche empiriche dovute alla raccolta condotta da Afanes'ev. Ma il vero nodo della discordia restava la storicità. Propp ricusò l'ipotesi di sviluppo di un sistema logico atemporale ribadendo la fondamentale successione delle funzioni, sempre identica in ogni fiaba, rimarcando come l'azione narrativa si può cogliere a pieno soltanto osservando lo sviluppo temporale delle funzioni della fiaba. Pertanto, concludeva Propp, non si può adoperare un sistema logico al posto di un criterio cronologico, pena lo snaturamento della sintassi della fiaba.

Dopo questa lunga disamina del modello proppiano e del dibattito con Lévi-Strauss, l'autore propone una revisione del sistema delle funzioni, proponendo di raggrupparlo in gruppi denominati «prove», come ad esempio (divieto-infrazione; investigazionedelazione; tranello-connivenza), sulla base di un modello fisso di domande e risposte. Inoltre alle trentuno funzioni individuate da Propp se ne aggiungono su proposta di Sanga altre due: Situazione iniziale e Sciagura. Al centro del libro si colloca, pertanto, l'idea dell'ampliamento delle funzioni (da trentuno a trentatré) e del loro raggruppamento in undici «prove». Questo approfondimento è corredato da esempi, tabelle e quadri sinottici riepilogativi.

8 Alla revisione della morfologia della fiaba segue una densa ricapitolazione degli studi delle precedenti stagioni otto-novecentesche, a partire da quelli di ambito filologicoletterario, dominanti negli studi folklorici. Sanga dichiara innanzitutto il debito della cultura italiana verso la preziosa opera letteraria di Calvino che venne vista con sospetto dagli studiosi di folklore per via della riscrittura in italiano, mentre, sottolinea l'autore, la sua operazione - non molto dissimile da quanto fatto dagli stessi folkloristi risulta coerente e cristallina poiché dichiarando le fonti originali permette di cogliere il contributo autoriale e letterario del curatore. Molto spesso il rifacimento dei testi per arricchirne l'aspetto stilistico-letterario era sottaciuto dai folkloristi e secondo un insigne studioso come Alessandro D'Ancona questo era anche un dovere implicito da adempiere per elevare quei poveri e rozzi testi. E infatti, di fronte alla prima raccolta di fiabe condotta con metodo stenografico da Vittorio Imbriani, non mancarono le rampogne di D'Ancona che biasimava la fedeltà filologica dell'autore. Grazie al magnetofono divenne possibile specializzarsi in trascrizioni fonetiche in grado di restituire il sapore dell'oralità nelle fiabe trascritte, mentre l'antropologia visuale e il documentario etnografico hanno aperto la strada - rimasta però inesplorata - della osservazione della prossemica e della gestualità del narratore in rapporto al suo uditorio. Un aspetto cruciale degli studi sulla fiabistica è, inoltre, quello linguistico che ha posto in evidenza l'esistenza di una koinè regionale della narrazione (e del canto) orale. Questa dimensione sovra-locale apre l'ultimo e decisivo terreno di riflessione dell'autore, quello inerente le forme di circolazione della fiaba. Se la ballata popolare si è diffusa grazie ai professionisti dello spettacolo di piazza come i cantastorie ed è stata poi tramandata grazie ai fogli volanti e alla trasmissione orale dei brani, la fiaba è stata 
veicolata nella memoria collettiva da figure specializzate, da narratori professionali o semi-professsionali delle classi marginali: vagabondi, mendicanti, girovaghi, ambulanti. Si tratta di una osservazione cruciale per Sanga che sottolinea su questo punto il debito contratto nei confronti del lavoro demologico di Bruno Pianta sui cantastorie, il pubblico e il mercato della musica e dei canti di tradizione orale. Una pista suggestiva e ancora da esplorare a fondo.

9 La riflessione sulle figure emblematiche della trasmissione orale della fiaba permette a Sanga di ritornare a Propp per formulare, anche in questo caso, una tesi originale. Non si tratta di forme decadute di antichi riti iniziatici delle società di pastori, raccoglitori e cacciatori, come ipotizzato da Propp - e anche su questo punto si aprì un dibattito con Lèvi-Strauss che considerava mito e fiaba come due ambiti complementari ma separati - ma della conseguenza dello scambio e del contatto tra due modi di produzione: cacciatori e coltivatori. Non in modo lineare, come sosteneva Propp, ma in modo simbiotico, mediato dalle figure dei marginali, che dei cacciatori-raccoglitori incarnano la prosecuzione ideale nelle società agricole, produttive e sedentarie. Non più predatori della natura, ma predatori di una società naturalizzata, che diventa la foresta da depredare. L'abilità mnemonica, fabulatoria e performativa del mondo marginale ha permesso alla fiaba di trasmettersi nelle realtà rurali e contadine. A conferma della sua ipotesi Sanga osserva le formule finali delle fiabe e i numerosi esempi riportati attestano il tono questuante assunto dalla chiusura dei racconti. Se i folkloristi hanno incontrato anziane donne contadine come depositarie della fiaba e delle tradizioni orali, va però considerato che tale patrimonio scaturito, in realtà, da una precedente fase storica, era stato messo in circolazione grazie all'opera dei professionisti delle classi marginali.

Discostandosi dagli approcci alla fiaba di tipo performativo, Sanga ha riportato al centro del discorso l'analisi dei testi, della morfologia della fiaba e delle forme di comunicazione e diffusione dell'oralità, offrendoci un prezioso strumento didattico che fa il punto su un tema classico per l'antropologia che, tuttavia, non ha perso il suo spazio nella nostra società. Infatti, seguire gli sviluppi della fiaba nel presente, dove tra l'altro è ben radicata, resta un compito da espletare attraverso nuove indagini empiriche sul mondo dei media e sulle forme di narrazione in ambito locale e familiare - tutt'altro che defunte e capaci di creare nuovi sincretismi - disponendo di uno strumento analitico così ampio ed esaustivo sulla fiaba magica tradizionale. Ci auguriamo, pertanto, che questo volume possa essere un punto di partenza decisivo per avviare una nuova fase negli studi sulla fiaba e la narrazione orale. 